\title{
PROCESSOS INFLAMATÓRIOS DA OBESIDADE
}

\author{
INFLAMMATORY PROCESSES OF OBESITY
}

\section{Crislene Sippel $\mathrm{a}^{*}$, Rafaela Mundstock de Azevedo Bastian ${ }^{\mathrm{b}^{*}}$, Janine Giovanella ${ }^{\mathrm{c}^{*}}$, Christiane Faccin $^{\mathrm{d}^{*}}$, Verônica Contini ${ }^{*}$, Simone Morelo Dal Bosco ${ }^{\mathrm{f}^{*}}$}

\author{
acrislenea@universo.univates.br, brafabastian@hotmail.com, cjaninegiovanella@hotmail.com, dchrisfaccin@hotmail.com, \\ everonica.contini@gmail, fsimonebosco@gmail.com \\ *Univates - Lajeado (RS), Brasil
}

\section{RESUMO}

Introduçáo: A prevalência da obesidade vem crescendo ano após ano em todo o mundo, já sendo considerada pela Organização Mundial da Saúde (OMS) como uma epidemia mundial. Dados brasileiros revelam que mais de $13 \%$ da população é obesa. Objetivo: Revisar a literatura acerca da interferência dos fatores inflamatórios na obesidade. Métodos: A revisão crítica foi elaborada a partir da compilação de artigos de revisão e estudos originais publicados entre 2010 e 2013, indexados em português e inglês nas bases de dados SciELO e PubMed. As palavras-chave utilizadas nas pesquisas foram obesidade, tecido adiposo, inflamaçáo, adipocinas e fatores de crescimento. Resultados: A compilação dos artigos selecionados esclarece e fortalece o conceito da obesidade como uma doença inflamatória, com maior expressão, em obesos, de adipocinas pró-inflamatórias como a interleucina-6, fator de necrose tumoralalfa, adipsina, angiotensinogênio, leptina, resistina e visfatina e menor expressão das adipocinas antiinflamatórias, interleucina-10, adiponectina e SFRP5. Além disto, os resultados demonstram que alguns fatores de crescimento também estão envolvidos no processo inflamatório da obesidade. Conclusão: Conclui-se que, com o grande número de artigos publicados em inglês e em português, a literatura científica hoje nos fornece uma melhor compreensão do processo inflamatório na obesidade, no entanto, muitos mecanismos envolvidos ainda precisam ser investigados e esclarecidos. Dessa forma, pesquisas nesse campo devem ser incentivadas para melhor embasamento das medidas de promoção à saúde, prevenção e tratamento da obesidade e suas comorbidades.

Palavras-chave: Obesidade, Inflamação, tecido adiposo, Nutrição.

\begin{abstract}
Objective: To review the literature on interference of inflammatory factors in obesity. Methods: The critical review was elaborated from the compilation of review articles and original studies published between 2010 and 2013, indexed in Portuguese and English in SciELO and PubMed databases. The keywords used for the researches were obesity, adipose tissue, inflammation, adipokines and growth factors. Results: The compilation of the selected articles clarifies and strengthens the concept of obesity as an inflammatory disease, with higher expression in obese of proinflammatory adipokines such interleukin-6, tumor necrosis factor-alpha, adipsin, angiotensinogen, leptin, resistin and visfatin and lower expression of inflammatory adipokines, interleukin-10, adiponectin and SFRP5. Furthermore, the results show that some growth factors are also involved in the inflammatory process of obesity. Conclusion: We conclude that, with the large number of articles published in English and Portuguese, the scientific literature today provides us with a better understanding of the inflammatory process in obesity, however, many mechanisms have to be
\end{abstract}


investigated and clarified. Thus, researches in this field should be encouraged for better basis of measures for health promotion, prevention and treatment of obesity and its comorbidities.

Keywords: Obesity, Inflammation, adipose tissue, Nutrition.

\section{Introdução}

A prevalência da obesidade vem crescendo ano após ano em todo o mundo, já sendo considerada pela Organização Mundial da Saúde (OMS) como uma epidemia mundial. Informaçóes do relatório World Health Statistics 2012, da OMS, apontam que a obesidade está presente em $12 \%$ da população mundial e é a causa morte de 2,8 milhóes de pessoas por ano. $\mathrm{O}$ continente americano é o responsável pela maior parcela de obesos do mundo, $26 \%$ da população adulta, enquanto apenas $3 \%$ da população do sudoeste asiático é obesa. Ainda, segundo a pesquisa, a obesidade dobrou em todas as regiōes do mundo entre os anos de 1980 e $2008^{1}$. Dados brasileiros revelam que mais de $13 \%$ da população é obesa $^{2}$.

$\mathrm{Na}$ década de 1990, a obesidade foi reconhecida como uma doença inflamatória, após um primeiro estudo, realizado com ratos, demonstra maior expressáo do gene codificador da adipocina pró-inflamatória, fator de necrose tumoral-alfa $(T N F-\alpha)$ no tecido adiposo, além de uma redução da sensibilidade à insulina após exposição a uma dieta de ganho de peso ${ }^{3}$. Desde então, outros estudos têm encontrado uma relaçáo entre a obesidade e a alteração das funçóes metabólicas e endócrinas do tecido adiposo, demonstrando que, em indivíduos obesos, o tecido adiposo aumenta a síntese de adipocinas (citocinas), com efeito pró-inflamatório, como o angiotensinogêneo, o $T N F$ - $\alpha$, a interleucina 6 (IL-6), a leptina e outras, ao contrário do observado em indivíduos magros, em que estas moléculas não são tão expressas ${ }^{4,5}$. A resposta inflamatória da obesidade leva não somente à elevação da expressão de adipocinas pró-inflamatórias, mas também à redução de adipocinas com propriedades anti-inflamatórias, como a adiponectina ${ }^{4}$. Porém, quando o excesso de peso corporal é eliminado, há uma maior expressão nas adipocinas anti-inflamatórias, uma redução das pró-inflamatórias e uma melhora na resposta à resistência à insulina ${ }^{5}$.

O tecido adiposo é o maior órgão do corpo humano e está subdividido em tecido adiposo branco, marrom e bege $e^{6,7}$. Até pouco tempo, acreditava-se que sua única função seria a de estocar energia. Atualmente, sabe-se que ele é responsável por inúmeras funções, tais como regulador da temperatura corporal, protetor mecânico ao trauma, estoque de energia, secreção de proteínas e peptídeos bioativos, sendo regulado por nervos, hormônios, nutrientes e mecanismos autócrinos e parácrinos.
O tecido adiposo é composto por adipócitos, responsáveis pela secreção de ácidos graxos, colesterol, retinol, hormônios esteroidais e alguns mediadores químicos envolvidos em processos inflamatórios ${ }^{6}$, que serão abordados em detalhes a seguir.

\section{Métodos}

A revisão crítica foi elaborada a partir da compilação de artigos de revisão e estudos originais publicados entre 2010 e 2013, indexados em português e inglês nas bases de dados SciELO e PubMed. As palavras-chaves utilizadas para as pesquisas foram obesidade, tecido adiposo, inflamaçáo, adipocinas e fatores de crescimento em português e obesity, adipose tissue, inflammation, adipokines e growth factors em inglês. Artigos e bases de dados anteriores a 2010, necessários para o esclarecimento de conceitos e confirmação de dados relativos à obesidade, também foram citados.

Uma busca foi realizada e encontramos 77 artigos com o tema pesquisado, dos quais selecionamos 54 que serviram para o embasamento teórico e para a explanação da presente revisão.

\section{Resultados e Discussão}

\section{Tecido adiposo}

O tecido adiposo é formado pelos tecidos visceral (TAV) e subcutâneo (TAS), os quais, devido à sua localização, possuem características metabólicas diferentes. O TAV, localizado principalmente na regiáo abdominal, é mais ativo metabolicamente, promove mais lipólise e liberação de ácidos graxos, possui mais resistência à insulina e secreta uma maior quantidade de adipocinas pró-inflamatórias (resistina, angiotensina I, resistina, PAI-1, PCR e IL-6). Por outro lado, o TAS, situado na região glúteo-femural e abdominal, produz uma maior quantidade de adipocinas pró-inflamatórias, como a leptina e a acylation stimulating protein (ASP) ${ }^{8}$.

\section{Tecido adiposo branco}

Situado em diversas regióes do organismo, tem as funçóes de armazenamento de energia, na forma de 
triglicerídeos, de manutenção da temperatura corporal, além de proteger contra choques mecânicos. Ele é secretor de moléculas bioativas, as adipocinas, substâncias biológicas que estão diretamente envolvidas em processos metabólicos e fisiológicos do organismo?. Os adipócitos correspondem à metade de sua composição e os demais $50 \%$ correspondem a tecido conjuntivo e nervoso, nódulos linfáticos, leucócitos, macrófagos, fibroblastos, pré-adipócitos e células mesenquimais ${ }^{10}$.

\section{Tecido adiposo marrom}

Também conhecido como baby fat ${ }^{11}$, acreditava-se que somente crianças e jovens possuíssem tecido adiposo marrom. Porém, estudos atuais, com a utilização de tomografia por emissão de pósitrons, revelaram que os adultos também o possuem e que sua expressão está aumentada após a exposição a baixas temperaturas durante determinado espaço de tempo ${ }^{12}$.

Este processo ocorre devido ao aumento da atividade nervosa simpática nos tecidos adiposos, por meio da ativação de alguns receptores transient receptor potential channels (TRP). A noradrenalina liberada pelo sistema nervoso simpático liga-se aos receptores $\beta$-adrenérgicos $(\beta A R)$ e iniciam a cascata de sinalização de hidrólise de triglicerídeos. Os ácidos graxos liberados ativam a proteína desacopladora 1 (UCP1), que será oxidada para servir como fonte de energia pelo corpo na forma de dissipação de calor. A ativação simpática crônica produz a hiperplasia do tecido adiposo marrom e também a indução de células bege, aumentando o gasto energético corporal e proporcionando a diminuição da gordura corpórea. A região do pescoço, da supraclavicular, do mediastino e da suprarrenal foram as regióes anatômicas em que o tecido teve maior expressão ${ }^{12}$.

A coloração escurecida do tecido se dá devido à presença da enzima citocromo oxidase dentro das mitocôndrias da célula ${ }^{11}$. Recentemente, descobriu-se que existem dois tipos distintos de gordura marrom: a clássica, descrita anteriormente, e outra linhagem, que se origina pela gordura branca denominada também de gordura bege?.

\section{Tecido adiposo bege}

Definidas como um "escurecimento" do tecido adiposo branco, estas células possuem um padrão de expressão gênica diferente da gordura branca e da marrom, apesar de apresentarem uma baixa expressão basal de UCP1, assim como o tecido branco, e responderem a estimulação do AMP cíclico, com altas taxas de expressão de UCP1, como o tecido marrom ${ }^{7}$. O tecido adiposo bege possui uma especial sensibilidade ao hormônio polipeptídeo irisina, liberado durante a prática de atividade física. $\mathrm{O}$ exercício físico aumenta a expressáo do receptor ativado por proliferador de peroxissoma 1-alfa (PGC-1 $\alpha$ ) no músculo ${ }^{7,13}$. O PGC-1 $\alpha$ protege contra o ganho de peso, inflamação, estresse oxidativo, perda de massa muscular e óssea e melhora a sensibilidade à insulina ${ }^{13}$. Em seguida, há o aumento da expressão da proteína fibronectin type III domain containing 5 (FNDC5), responsável por aumentar a secreçáo de irisina na circulação. A irisina, então, transforma o tecido adiposo branco em tecido adiposo marrom por meio de sua ligação a um receptor desconhecido nos adipócitos brancos, induzindo a expressáo de peroxisome proliferator-activated receptor gamma (PPARG), elevando a expressáo da UCP1, que provoca o aumento do gasto energético, promovendo efeitos favoráveis sobre o metabolismo e resistência a doenças metabólicas ${ }^{7}$.

\section{Adipocinas inflamatórias}

\section{Interleucina 6 (IL-6) e Fator de necrose tumoral alfa (TNF- $\alpha)$}

O sistema imunológico é constituído por uma complexa rede de células e moléculas dispersas por todo o organismo e se caracteriza pela capacidade de reconhecer determinadas estruturas moleculares ou antígenos. Desenvolve uma resposta efetora diante destes estímulos, provocando a sua destruição ou inativação ${ }^{14}$. As proteínas do sistema imunológico representam 20 a $25 \%$ da concentração total de proteínas plasmáticas e o seu componente celular representa aproximadamente $15 \%$ das células corporais ${ }^{14}$.

As adipocinas inflamatórias são proteínas de baixo peso molecular, com diversas funçóes metabólicas e endócrinas, que participam da inflamação e resposta do sistema imune. São consideradas como fatores de risco independentes para doenças da artéria coronária e cerebrovascular. As principais fontes de adipocinas sáo os tecidos adiposos subcutâneo e visceral ${ }^{15}$.

O tecido adiposo não é apenas um órgão de estoque. Tem papel como produtor de certas substâncias bioativas como a IL-6, com funçóes inflamatórias ${ }^{16}$. Com o aumento da quantidade das células adiposas ocorre uma alteração na produção de adipocinas inflamatórias, ou seja, quanto maior a quantidade de tecido adiposo maior é a secreção dessas adipocinas, como TNF- $\alpha$ e IL6 ${ }^{15}$. A IL-6 e o TNF- $\alpha$ são adipocinas com função imunológica, e são produzidas pelos adipócitos em resposta a estímulos infecciosos ou inflamatórios ${ }^{14}$. 
Modelos experimentais sugerem o envolvimento de diversas adipocinas, principalmente IL-6 e TNF- $\alpha$ no dano isquêmico cerebral. O TNF- $\alpha$ e a interleucina- $1 \beta$ (IL-1ß) encontram-se elevados após isquemia cerebral, podendo induzir uma reaçáo inflamatória no SNC, junto com a IL-6. Além disso, parecem modular diretamente o processo de apoptose das células no SNC, promovendo diferenciação, proliferação e subsequente infiltração leucocitária ${ }^{17}$.

As adipocinas têm sido implicadas em diversos mecanismos, que podem potencializar a lesão isquêmica cerebral. Isso porque ocorre a liberação da enzima óxido nítrico sintetase pelos astrócitos, o recrutamento, a ativação e a adesão leucocitárias ao endotélio, a promoção do estado pró-coagulante endotelial e a regulaçáo do processo de apoptose. A interleucina-6 e o TNF- $\alpha$ são adipocinas que participam ativamente dos mecanismos de lesão cerebral ${ }^{17}$.

Estudos sugerem que uma das mais importantes descobertas das pesquisas recentes em obesidade é o conceito de que ela é caracterizada por uma inflamação crônica. Dentre todas as adipocinas, sem dúvida, a IL-6, o TNF- $\alpha$, a leptina (pró-inflamatórias) e a adiponectina (anti-inflamatória) vêm recebendo atençáo especial. $\mathrm{O}$ aumento da concentração das adipocinas pró-inflamatórias promove grande impacto em diversas funçóes corporais que estão fortemente correlacionadas com doenças cardiovasculares ${ }^{17}$.

Em um estudo realizado com mulheres obesas submetidas a um tratamento multidisciplinar, composto por exercício físico, dieta e acompanhamento psicológico, observou-se redução de cerca de $10 \%$ da massa corporal inicial. Essa redução foi associada com menores concentraçóes de adipocinas pró-inflamatórias e maiores de adiponectina. Os autores concluíram que a redução da massa corporal é um método seguro para diminuir o estado inflamatório e modular na disfunção endotelial dessas mulheres obesas ${ }^{18}$.

A resposta inflamatória promove, por um lado, o aumento da síntese de diversas adipocinas com ação pró-inflamatória e, por outro, a redução da concentração plasmática de adiponectina, que apresenta ação anti-inflamatória. A secreção de IL-6 pelo tecido adiposo pode representar cerca de $10 \%$ a $30 \%$ dos teores circulantes dessa citocina, e o tecido adiposo visceral produz e secreta três vezes mais IL-6 do que o subcutâneo ${ }^{19}$. O conteúdo plasmático aumentado de IL-6 poderia estimular a síntese hepática de triacilglicerol, contribuindo para a hipertrigliceridemia associada à obesidade visceral ${ }^{20}$. Ela se liga aos receptores de IL-6R e ativa a cascata de sinalização intracelular, conduzindo à resposta inflamatória. Após isso, inibe a atividade da lipoproteína lipase e estimula lipólise, que afeta o perfil lipídico e pode contribuir para a patogênese da doença aterosclerótica ${ }^{21}$.
Há indícios recentes de que a IL-6 exerça ação direta sobre a sensibilidade à insulina, alterando a sinalização insulínica em hepatócitos mediante a inibição do receptor de insulina dependente de autofosforilação, promovendo, desse modo, resistência à ação do hormônio no tecido. A IL-6 pode agir de formas distintas, dependendo da sua concentração orgânica, tanto nos tecidos periféricos quanto no sistema nervoso central, influenciando o peso corporal, a homeostase energética e a sensibilidade insulínica ${ }^{20}$.

Outra citocina inflamatória importante é o TNF- $\alpha$. É produzida por adipócitos e está aumentada no tecido adiposo de roedores obesos, assim como em humanos. É uma citocina pró-inflamatória produzida pelos tecidos muscular, adiposo e linfoide e está envolvida com resistência à insulina por inibir a fosforilação da tirosina presente no substrato-1 do receptor de insulina (IRS-1). Em um estudo com ratos obesos, demonstrou-se também que a neutralização dos efeitos do TNF- $\alpha$ provocou aumento da sensibilidade insulínica ${ }^{22}$.

Há ainda referências de que triacilgliceróis e ácidos graxos livres exerçam forte influência na indução da expressão de TNF- $\alpha$. Em um estudo com roedores tratados com dieta hiperlipídica, verificou-se um aumento significativo da expressão de TNF- $\alpha$ e alteração da via de sinalização insulínica in vivo ${ }^{20}$.

\section{Adipsina}

A adipsina, assim como a IL-6, o TNF- $\alpha$ e os fatores do complemento B e C3, é uma adipocina com função imunológica ${ }^{23}$. Foi uma das primeiras proteínas produzidas pelo tecido adiposo, identificadas em meados de $1980^{6}$. Conhecida também como fator D, é por meio de sua interação com os complementos C3 e B que se origina a proteína estimuladora de acilação (ASP). Este fator estimula a síntese de triglicerídeos (TG) no tecido adiposo branco por aumentar a translocação de GLUT-4, a produção de glicerol-3-fosfato e a atividade da enzima catalisadora da síntese de diacil-glicerol aciltransferase (DGAT) e, além disso, ASP inibe a lipólise pela inibição da lipase hormônio-sensível (LHS) ${ }^{24}$.

Um estudo realizado com 35 pacientes que se submeteram a cirurgia plástica obteve 70 amostras de tecido adiposo branco (35 do depósito subcutâneo e 35 do visceral) para análise pelo método ELISA dos níveis de adipsina e outras substâncias. Os resultados demonstraram que os níveis de adipsina estavam aumentados no tecido adiposo subcutâneo, ao comparar com o tecido adiposo visceral. Notou-se, também, que a presença da adipsina era maior no sangue periférico do que no tecido adiposo branco ${ }^{25}$. 
A adipsina é produzida pelos adipócitos em resposta a estímulos infecciosos ou inflamatórios. Assim como as demais adipocinas com função imunológica, é uma molécula de sinalização considerada mediadora pró-inflamatória ${ }^{6}$. A presença de ASP e sua correlação positiva à adipsina foram evidenciadas em um estudo realizado por MacLaren et al. ${ }^{26}$, que sugerem ainda que a obesidade seria uma resposta compensatória ao aumento dos níveis de ASP.

\section{Angiotensinogênio (AGT)}

O angiotensinogênio é uma proteína circulante produzida, principalmente, pelo fígado, que faz parte do sistema renina-angiotensina-aldosterona. Ao ser clivado pela enzima renina, forma a angiotensina I, que, por sua vez, é clivada pela Enzima Conversora de Angiotensina (ECA), dando origem a angiotensina II, e esta possui diversas funções fisiológicas relacionadas ao aumento da pressão arterial, principalmente por meio da vasoconstrição arteriolar e por estimular a secreção da aldosterona ${ }^{27}$.

É uma adipocina envolvida na regulação da pressão arterial que, assim como as demais moléculas do sistema renina-angiotensina-aldosterona e o PAI-1, está no grupo das adipocinas com funçóes cardiovasculares. $\mathrm{O}$ angiotensinogênio vem sendo relacionado ao acúmulo de gordura corporal e sua participação estaria na diferenciação de adipócitos (estimulação da produção de prostaciclinas), sugerindo uma maior secreçáo no tecido adiposo visceral ${ }^{24}$. Estudos in vitro mostraram que a Ang II estimula a lipogênese e secreção de adipocinas pró-inflamatórias em adipócitos ${ }^{28}$.

Os efeitos metabólicos do sistema renina-angiotensina-aldosterona foram associados, em modelos animais, à eliminação sistemática de certos componentes: angiotensinogênio, renina, ACE, AT1 ou AT2 29 . O silenciamento do gene AGT reduziu significativamente a expressão de adipocinas pró-inflamatórias, incluindo a IL-6, TNF- $\alpha$, e proteína quimiotática de monócitos-1 $(\mathrm{MCP}-1)^{30}$.

\section{Leptina}

A descoberta de leptina em ratos obesos, em 1994, revolucionou a compreensão da biologia da obesidade na época. Ela está relacionada com a regulação da ingestão alimentar e gasto energético por meio da regulação pelo sistema nervoso central (SNC) ${ }^{31}$. Possui, também, funçốes sobre os linfócitos- $T$, atuando na proliferação de células envolvidas na resposta imune, gerando produção de linfocinas pró-inflamatórias, sendo, portanto, mediadora do estado nutricional e sobre a função imunológica ${ }^{32}$.

No metabolismo lipídico, a leptina está envolvida no aumento da oxidação lipídica pelo músculo esquelético e na redução da síntese de triglicerídeos no fígado. Além disso, possui efeitos sobre a fertilidade, angiogênese, controle da pressão sanguínea e na osteogênese ${ }^{33}$.

Em um estudo recente, foi demonstrada uma correlação positiva entre os níveis plasmáticos da adipocina leptina e o IMC, em homens, e de leptina com a prega cutânea triciptal (PCT) em mulheres. Ainda, observouse que os níveis de leptina entre indivíduos resistentes à insulina foram praticamente o dobro em comparação com os indivíduos com sensibilidade à insulina normal, com os mesmos níveis de adiposidade corporal. Também foi observada diferença significativa entre os valores de HOMA-IR e as concentraçóes da adipocina tanto em homens quanto em mulheres, sugerindo que a leptina é um importante preditor de resistência à insulina e outros riscos metabólicos, independentemente do nível de obesidade ${ }^{34}$.

\section{Resistina}

Outra adipocina pró-inflamatória, a resistina, pertencente à família das moléculas resistin-like ${ }^{35}$, é secretada por monócitos e adipócitos e expressa em indivíduos magros, porém indivíduos obesos comumente possuem níveis elevados da adipocina. Promove resistência insulínica, diminuindo a gliconeogenese hepática e tem ação regulatória da adipogênese devido à sua alta expressão nos pré-adipócitos ${ }^{36}$. É considerada mais do que um simples marcador de inflamação, uma vez que pode induzir a expressão do TNF- $\alpha$, da IL-6 e da interleucina 12 (IL-12) $)^{37}$. Em um estudo recente, observaram-se valores séricos de resistina significativamente mais elevados em mulheres com maior perímetro abdominal. Porém, nos indivíduos do gênero masculino, não foram encontradas diferenças nos valores sanguíneos da adipocina entre os participantes com excesso de peso e aqueles com peso considerado normal ${ }^{38}$.

\section{Visfatina}

Esta adipocina é secretada pela gordura visceral do tecido adiposo branco, sendo expressa pelos macrófagos destes ${ }^{39}$, em problemas de saúde como doença pulmonar aguda, artrite reumatoide, doença inflamatória intestinal e renal, e infarto agudo do miocárdio. Em humanos, a adipocina induz a produçáo de IL-6, TNF- $\alpha$ e IL-1 $\beta^{40}$, influencia a regulação da homeostase glicêmica por sua ligação ao receptor da insulina. Contudo, 
não competem com o hormônio por possuírem sítios diferenciados ${ }^{41}$.

Estudos sugerem que os níveis circulantes e a expressão da visfatina estão significativamente aumentados em mulheres com obesidade mórbida, quando comparadas a mulheres com peso corporal normal. Os níveis plasmáticos da adipocina também foram positivamente correlacionados com valores séricos das transaminases (aspartato aminotransferase, alanina aminotransferase, gama-glutamil transferase e fosfatase alcalina), de IL-6 e da proteína $\mathrm{C}$ reativa $(\mathrm{PCR})^{42}$. Em resumo, estudos sugerem que a visfatina pode desempenhar um papel na inflamação vascular e na aterosclerose ${ }^{43,44}$.

\section{Adipocinas anti-inflamatórias}

\section{Interleucina 10 (IL-10)}

A IL-10 é uma citocina anti-inflamatória capaz de inibir a síntese de adipocinas pró-inflamatórias por linfócitos $\mathrm{T}$ e macrófagos. Ela inibe a síntese do TNF, IL-1, IL-6 e IL-8 em monócitos e macrófagos e sua presença tem sido demonstrada em placas ateroscleróticas. Observou-se em estudos com animais que níveis baixos de IL-10 levaram ao desenvolvimento de lesóes ateroscleróticas morfologicamente maiores e mais instáveis. As evidências disponíveis sugerem um potencial papel protetor de IL-10 no desenvolvimento de aterosclerose ${ }^{45}$.

Vários mecanismos têm sido propostos para explicar a forma com que a IL-10 consiga inibir a síntese de adipocinas pró-inflamatórias. Um dos mais estudados é a inibição da ativação do fator de transcrição nuclear NF- $\kappa \beta$ por IL-10 em monócitos e células T ${ }^{45}$.

Um estudo realizado com ratos machos Wistar, submetidos à ligadura da artéria coronária esquerda, submeteu os animais à análise ecocardiográfica e os dividiu em grupos experimentais "falso operado (Sham)" e "IM (infarto do miocárdio)". Os resultados demonstraram que o grupo de animais submetidos ao IM apresentou aumento significativo de $26,5 \%(\mathrm{p}<0,05)$ na produção de TNF- $\alpha$ no músculo sóleo, quando comparado ao grupo Sham (falso operado) ${ }^{46}$.

\section{Adiponectina (AdipoQ)}

A AdipoQ, expressa em adipócitos diferenciados, é uma das adipocinas mais abundantes do plasma, representando de $0,01 \%$ a $0,05 \%$ do total de proteína plasmática ${ }^{47}$. Considerada protetora para doença cardiovascular, atenuando a progressão da aterosclerose, ela ainda melhora a sensibilidade à insulina, possui ação anti-inflamatória reduzindo os níveis séricos das adipocinas pró-inflamatórias IL-6 e TNF- $\alpha$, contribui na homeostase pós-prandial da glicose e de lipídeos e provoca o aumento da oxidação de gorduras ${ }^{9,47}$.

Estudos sugerem que baixos níveis plasmáticos da adipocina estejam correlacionados com o estabelecimento da $\mathrm{SM}^{48}$, uma vez que indivíduos obesos apresentam níveis plasmáticos de AdipoQ menores em comparação a indivíduos com peso adequado ${ }^{49}$, assim como sua expressão é igualmente reduzida na gordura visceral (obesidade intra-abdominal) $^{50}$. Níveis plasmáticos de LDL-colesterol e triglicerídeos também possuem correlação negativa com os valores circulantes da adipocina?. Além disso, as mulheres possuem valores mais elevados de adiponectina do que os homens ${ }^{50}$. O emagrecimento parece estar associado com a redução dos níveis de AdipoQ, na melhora da resposta à insulina e na regulação do HDL-colesterol ${ }^{51}$.

\section{SFRP5}

A secreted frizzled-related protein 5 ou SFRP5, pertencente à família SFRP, atua como moduladora das vias de sinalização wingless (Wnt) ${ }^{52}$. Adipocina com efeito anti-inflamatório é altamente expressa na retina, moderadamente no fígado e pouco expressa em indivíduos obesos e portadores de diabetes mellitus tipo 2 $(\mathrm{DM} 2)^{53}$. Além disso, a circulação sanguínea da SFRP5 está correlacionada com marcadores de adiposidade, como o IMC, relação cintura/quadril, percentual de gordura corporal, perfil lipídico, adiponectina e na resistência à insulina ${ }^{54}$.

Em um estudo recente envolvendo indivíduos obesos e eutróficos, com controle de idade e sexo, observou-se por parte dos participantes obesos, após a adesão a uma dieta hipocalórica durante 3 meses, redução de peso e melhora significativa nos níveis séricos de HOMA e na razão leptina/adiponectina, associadas com um aumento significativo de SRP5, levando a uma melhora na sensibilidade à insulina, sugerindo que este possa ser um novo sistema regulador da inflamação de baixo grau de obesidade, podendo ser diretamente influenciado por terapia nutricional adequada ${ }^{55}$.

\section{Fatores de crescimento}

Os fatores de crescimento vêm sendo associados ao tecido adiposo branco e são considerados adipocinas com diferentes funçóes neste complexo processo. $\mathrm{O}$ vascular endotelial growth factor (VEGF), fator de crescimento vascular endotelial, estimula a proliferação vascular no tecido adiposo branco; o transforming growth 
factor $\beta$ (TGF- $\beta$ ), fator de crescimento transformador $\beta$, além da proliferação dos pré-adipocitos, regula a diferenciaçáo, desenvolvimento e apoptose dos adipócitos; o insulin-like growth factor 1 (IGF-1), fator de crescimento do tipo insulina 1, estimula a proliferaçáo e diferenciação dos adipócitos e o hepatocyte growth factor (HGF), fator de crescimento de hepatócito, estimula a diferenciação e o desenvolvimento de adipócitos? .

Há evidência de desregulação do IGF-1 em casos de obesidade, sendo sugerida uma relação inversa entre os níveis totais de IGF-1 e IMC ${ }^{56}$. Em um estudo longitudinal com 48 pacientes portadores de fibromialgia, sendo nove pacientes magras, 26 com sobrepeso e 13 obesas, observou-se que os níveis totais de IGF-1 eram mais altos entre as participantes magras ${ }^{57}$.

Kaess et $a .^{58}$, em um estudo realizado com 1.806 participantes, observaram correlaçóes positivas entre os depósitos de gordura visceral, subcutâneo e periaórtica, e os níveis de VEGF e HGF. Foram avaliadas, ainda, diferenças entre os gêneros e encontrou-se forte associação da gordura visceral e periaórtica com os níveis de HGF em mulheres. Níveis significativamente mais elevados de VEGF, a partir de tecido adiposo visceral, foram observados em pacientes obesos em comparação com os não obesos ${ }^{59}$.

\section{Conclusão}

Conclui-se que indivíduos com excesso de peso apresentam maior secreção de adipocinas pró-inflamatórias e menor das anti-inflamatórias, caracterizando a obesidade como uma inflamação crônica, o que promove grande impacto em diversas funções corporais que estão fortemente correlacionadas a doenças cardiovasculares, resistência insulínica, hipertrigliceridemia e hipercolesterolemia.

No entanto, muitos mecanismos envolvidos no processo inflamatório da obesidade ainda precisam ser investigados e esclarecidos. Dessa forma, pesquisas nesse campo devem ser incentivadas para melhor embasamento das medidas de promoçáo à saúde, prevençáo e tratamento da obesidade e suas comorbidades.

\section{Referências}

1. World Health Organization. World Health Statistics. 2012: 35-6.

2. Instituto Brasileiro de Geografia e Estatística. Pesquisa de Orçamentos Familiares, 2008-2009: desnutrição cai e peso das crianças brasileiras ultrapassa padrão internacional. [citado em: 15 out. 2012]. Disponível em: http:// ibge.gov.br/home/presidencia/noticias/noticia_vizualiza. php?id-noticia $=1699$ \&id_pagina $=1$
3. Hotamisligil GS, Shargill NS, Spiegelman BM. Adipose expression of tumor-necrosis-factor-alpha-direct role in obesity-linked insulin resistance. Science. 1993; 259(5091): 87-91.

4. Bullo M, Casas-Agustench P, Migo-Correig P, Aranceta J, Salas Salvado J. Inflammation, obesity and comorbidities: the role of diet. Public Health Nutr. 2007; 10(10A): 1164-72.

5. Shah A, Mehta N, Reilly MP. Adipose inflammation, insulin resistance, and cardiovascular disease. JPEN J Parenter Enteral Nutr. 2008; 32(6): 638-44.

6. Prado WL, Lofrano MC, Oyama LM, Damaso AR. Obesidade e adipocinas inflamatórias: implicaçóes práticas para a prescrição de exercício. Rev Bras Med Esporte. 2009; 15(5): 378-83.

7. Boström P, Wu J, Jedrychowski MP, Korde A, Ye L, Lo JC, et al. A PGC1 $\alpha$-dependent myokine that drives browning of white fat and thermogenesis. Nature. 2012; 481(7382): 463-8.

8. Porter SA, Massaro JM, Hoffman U, Vasan RS, O’Donnel CJ, Fox CS. Abdominal subcutaneous adipose tissue: a protective fat depot? Diabetes Care. 2009; 32: 1068-75.

9. Fonseca-Alaniz MH, Takada J, Alonso-Vale MIC, Lima FB. $\mathrm{O}$ tecido adiposo como centro regulador do metabolismo. Arq Bras Endocrinol Metab. 2006; 50(2): 216-29.

10. Ahima RS, Flier JS. Adipose tissue as an endocrine organ. Trends Endocrinol Metab. 2000; 11: 327-32.

11. Cannon B, Nedergaard J. Brown adipose tissue: function and physiological significance. Physiol Rev. 2004; 84: 277-359.

12. Saito M. Brown adipose tissue as a regulator of energy expenditure and body fat in humans. Diabetes Metab J. 2013; 37: 22-9.

13. Handschin C, Spiegelman BM. The role of exercise and PGC1alpha in inflammation and chronic disease. Nature. 2008; 454: 463-9.

14. Martínez AC, Alvarez-Mon M. El sistema inmume (I): Conceptos generales, adaptaciónal, ejercício físico e implicaciones clínicas. Arch Med Deporte. 1999; 69: 47-54.

15. Carvalho MHC, Colaço AL, Fortes ZB. Citocinas, disfunçấo endotelial e resistência à insulina. Arq Bras Endocrinol Metab; 2006.

16. Fernández-Sánchez A, Madrigal-Santillán E, Bautista $M$, Esquivel-Soto J, Morales-Gonzáles A, Esquivel-Chirino $\mathrm{C}$, et al. Inflammation, oxidative stress and obesity. International Journal of Molecular Sciences. 2011; 12(5): 3117-32.

17. Souza JR, Oliveira RT, Blotta MH, Coelho OR. Níveis séricos de interleucina-6 (IL-6), interleucina-18 (IL-18) e proteína $\mathrm{C}$ reativa (PCR) na síndrome coronariana aguda sem supradesnivelamento do ST em pacientes com diabete tipo 2. Arq Bras Cardiol. 2008; 90(2): 94-9.

18. Ziccardi P, Nappo F, Giugliano G, Esposito K, Marfella $\mathrm{R}$, Cioffi $\mathrm{M}$, et al. Reduction of inflammatory cytokine 
concentrations and improvement endothelial functions in obese women after weight loss over one year. Circulation. 2002; 105: 804-9.

19. Steemburgo T, Azevedo MJ, Martínez JA. Interação entre gene e nutriente e sua associação à obesidade e ao diabetes melito. Arq Bras Endocrinol Metab, São Paulo. 2009; 53(5): 497-508.

20. Guimarães DED, Sardinha FLC, Mizurini DM, Carmo MGT. Adipocitocinas: uma nova visão do tecido adiposo. Rev Nutr. 2007; 20(5): 549-59.

21. Van Hall G, Steensberg A, Sacchetti M, Fischer C, Keller C, Schjerling P, et al. Interleukin-6 stimulates lipolysis and fat oxidation in humans. J Clin Endocrinol Metab. 2003; 88(7): 3005-10.

22. Montani JP, Antic V, Yang Z, Dulloo A. Pathways from obesity to hypertension: from the perspective of a vicious triagle. Int J Obesity. 2002; 26: S28-S38.

23. Barbosa VS, Rego JS, Nílzio A. Possível papel das adipocinas no lúpus eritematoso sistêmico e na artrite reumatoide. Rev. Bras. Reumatol. [on-line]. 2012; 52(2): 278-87.

24. Fonseca-Alaniz, MH, Takada J, Alonso-Vale MI, Lima FB. O tecido adiposo como órgáo endócrino: da teoria à prática. J. Pediatr, Rio de Janeiro. [on-line]. 2007; 83(5): S192-S203.

25. Blogowski W, Budkowska M, Salata D, Serwin K, Dolegowska B, Lokaj M, et al. Clinical analysis of selected complement-derived molecules in human adipose tissue. J Transl Med. 2013; 11: 11.

26. Maclaren RE, Cui W, Lu H, Simard S, Cianflone K. Association of adipocyte genes with ASP expression: a microarray analysis of subcutaneous and omental adipose tissue in morbidly obese subjects. BMC Med Genomics; 2010.

27. Levy MN, Koeppen BM, Stanton BA. Fundamentos de fisiologia. Tradução de Nephtali Segal Grinbaum [et al.]. 4 ed. Rio de Janeiro: Elsevier; 2006.

28. Kalupahana NS, Massiera F, Quignard-Boulange A, Ailhaud G. Overproduction of angiotensinogen from adipose tissue induces adipose inflammation, glucose intolerance, and insulin resistance. Obesity (Silver Spring). 2012; 20: 48-56.

29. Kalupahana N S, Moustaid-Moussa N. The renin-angiotensin system: a link between obesity, inflammation and insulin resistance. Obes Rev. 2012; 13: 136-49.

30. Carroll WX, Kalupahana NS, Booker SL, Siriwardhana N, LeMieux M, Saxton AM, et al. Angiotensinogen gene silencing reduces markers of lipid accumulation and inflammation in cultured adipocytes. Front Endocrinol (Lausanne). 2013; 4: 10.

31. Zhang Y, Proença R, Maffie M, Barone M, Leopold L, Friedman JM. Positional cloning of the mouse obese gene and its human homologue. Nature. 1994; 372: 425-32.

32. Lord GM, Matarese G, Howard JK, Baker RJ, Bloom SR, Lechler RI. Leptin modulates the T-cell immune response and reverses starvation-induced immunosuppression. Nature. 1998; 394: 897-901.
33. Fruhbeck G, Gomez-Ambrosi J, Muruzábal FJ, Burrell MA. The adipocyte: a model for integration of endocrine and metabolic signaling in energy metabolism regulation. Am J Physiol Endocrinol Metab. 2001; 280: E827-47.

34. Zuo H, Shi Z, Yuan B, Dai Y, Wu G, Hussain A. Association between serum leptin concentrations and insulin resistance: a population-based study from China. Plos One. 2013; 8(1): e54615.

35. Kim KW, Shin YH, Lee KE, Kim ES, Sohn MH, Kim KE. Relationship between adipokines and manifestations of childhood asthma. Pediatr Allergy Immunol. 2008; 19: 535-40.

36. Mcternan PG, Mcternan CL, Chetty R, Jenner K, Fisher $\mathrm{FM}$, Lauer MN, et al. Increased resistin gene and protein expression in human abdominal adipose tissue. J Clin Endocrinol Metabol. 2002; 87: 2407-10.

37. Larochelle J, Freiler J, Dice J, Hagan L. Plasma resistin levels in asthmatics as a marker of disease state. J Asthma. 2007; 44: 509-13.

38. Loureiro C, Pinto AM, Muc M, Pereira SV. Valores de resistina, adiponectina e leptina em doentes com asma e excesso de peso. Rev Port Imunoalergologia. 2012; 20(2): 121-8.

39. Antuna-Puente B, Feve B, Fellahi S, Bastard JP. Adipokines: the missing link between insulin resistance and obesity. Diabetes Metab. 2008; 34(1): 2-11.

40. Moschen AR, Kaser A, Enrich B, Mosheimer B, Theurl $\mathrm{M}$, Niederegger $\mathrm{H}$, et al. Visfatin and adipocytokine with pro-Inflammatory and immunomodulating properties. J Immunol. 2007; 178: 1748-58.

41. Fukuhara A, Matsuda M, Nishizawa M, Segawa K, Tanaka M, Kishimoto K, et al. Visfatin: a protein secreted by visceral fat that mimics the effects of insulin. Science. 2005; 307: 426-30.

42. Ledantes JAP. Visfatina: un potencial biomarcador de esteatohepatitis no alcohólica en mujeres con obesidad mórbida no diabéticas [tese]. Reus-Tarragona, Espanha: Universitat Rovira I Virgili; 2013.

43. Romacho T, Azcutia V, Vazquez-Bella M, Matesanz N, Cercas E, Nevado J, et al. Extracellular PBEF/NAMPT/ visfatin activates proinflammatory signalling in human vascular smooth muscle cells through nicotinamide phosphoribosyltransferase activity. Diabetologia. 2009; 52: 2455-63.

44. Adya R, Tan BK, Chen J, Randeva HS. Pre-B cell colony enhancing factor (PBEF)/visfatin induces secretion of MCP-1 in human endothelial cells: role in visfatin induced angiogenesis. Atherosclerosis. 2009; 205: 113-9.

45. Fernández RP, Kaski JC. Interleucina-10 y enfermedad coronaria. Rev Esp Cardiol. 2002; 55(7): 738-50.

46. Lopes RD, Batista-Júnior ML, Rosa JC, Lira FS, Martins Jr E, Shimura AY, et al. Alteraçóes na produção de IL-10 e TNF- $\alpha$ no músculo esquelético em ratos com insuficiência cardíaca secundária ao infarto do miocárdio. Arq Bras Cardiol; 2010; 94(3): 313-20.

47. Berg AH, Combs TP, Scherer PE. ACRP30/adiponectin: an adipokine regulating glucose and lipid metabolism. Trends Endocrinol Metab. 2002; 13: 84-9. 
48. Funahashi T, Matsuzawa Y, Kihara S. Adiponectin as a potential key player in metabolic syndrome insights into atherosclerosis, diabetes and cancer. Int Congress Series. 2004; 1262: 368-71.

49. Weyer C, Funahashi T, Tanaka S. Hypoadiponectinemia in obesity and type 2 diabetes: close association with insulin resistance and hyperinsulinemia. J Clin Endocrinol Metab. 2001; 86: 1930-5.

50. Nop M, Havel PJ, Utzschneider KM, Carr DB, Sinha MK, Boyko EJ, et al. Relationship of adiponectin to body fat distribution, insulin sensitivity and plasma lipoproteins: evidence for independent roles of age and sex. Diabetologia. 2003; 46: 459-69.

51. Geloneze B, Pereira JA, Pareja JC, Lima MMO, Lazarin MACT, Souza ICP, et al. Reversão da síndrome metabólica na obesidade mórbida: adiponectina como um marcador da sensibilização à insulina e do aumento do HDL-colesterol após o bypass gástrico. Arq Bras Endocrinol Metab. 2009; 53(2): 293-300.

52. SFRP5 secreted frizzled-related protein 5 [Homo sapiens (human)]. Gene ID: 6425, updated on 10-Mar-2013. [citado em: 02 abr. 2013]. Disponível em: http://www.ncbi. nlm.nih.gov/gene/6425

53. Ouchi N, Higuchi A, Ohashi K, Oshima Y, Gokce N, Shibata R, et al. Secreted frizzled related protein 5 is an anti-inflammatory adipokine that modulates metabolic dysfunction in obesity. Science. 2010; 329: 454-7.
54. Hu W, Li L, Yang M, Luo X, Ran W, Liu D, et al. Circulating Sfrp5 is a signature of obesity-related metabolic disorders and is regulated by glucose and liraglutide in humans. J Clin Endocrinol Metab. 2013, Jan; 98(1): 290-8. doi: 10.1210/ jc.2012-2466.

55. Schulte DM, Müller N, Neumann K, Oberhäuser F, Faust M, Güdelhöfer H, et al. Pro-inflammatory wnt5a and anti-inflammatory SFRP5 are differentially regulated by nutritional factors in obese human subjects. Plos One. 2012; 7(2): e32437.

56. Friedrich N, Jorgensen T, Juul A, Spielhagen C, Nauck M, Wallaschofski $\mathrm{H}$, et al. Insulin-like growth factor I and anthropometric parameters in a Danish population. Exp Clin Endocrinol Diabetes. 2012; 120:171-4.

57. Bjersing JL, Erlandsson AM, Bokarewa MI, Mannerkorp K. Exercise and obesity in fibromyalgia: beneficial roles of IGF-1 and resistin? Arthritis Research \& Therapy; 2013.

58. Kaess BM, Pedley A, Massaro JM, Larson MG, Corsini E, HoffmannU, et al. Relation of vascular growth factors with ct-derived measures of body fat distribuition: the Framingham heart study. J Clin Endocrinol Metab, 2012 Mar; 97(3): 987-94.

59. Lysaght J, Van Der Stock EP, Allott EH, Casey R, Donohoe CL, Howard JM, et al. Pro-inflammatory and tumour proliferative properties of excess visceral adipose tissue. Cancer Lett. 2011 Dec;15; 312(1): 62-72. 